\title{
INFOSOCIABILIDAD: MONITORIZACIÓN E INVESTIGACIÓN EN LA WEB 2.0 PARA LA TOMA DE DECISIONES
}

\author{
Miguel Del-Fresno-García
}

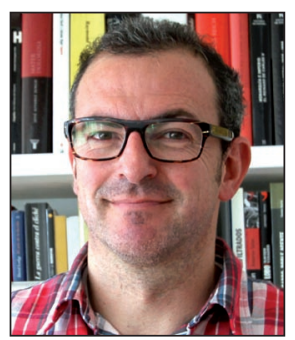

Miguel Del-Fresno-García es profesor en la UNED, doctor en sociología (UNED), DEA y master en sociedad de la información y el conocimiento (UOC), executive master en e-business (Instituto de Empresa), MBA (Instituto de Empresa) y licenciado en filosofía (UCM). Ha trabajado como profesor tutor de técnicas de investigación social (trabajo social) y etnografía (antropología) en la UNED, profesor de marketing (ADE) en la Universidad Camilo José Cela y de marketing y user experience design en el Instituto Europeo de Diseño. En su faceta profesional, es CEO de QUOR 2.0, empresa dedicada a la investigación de reputación online y comunicación 2.0. Anteriormente ha sido director de marketing y comunicación en Elsevier, director de marketing y comunicación de la cadena de librerías de Casa del Libro (Planeta) y director de marketing y miembro del equipo fundador de casadelibro.com. Como consultor ha trabajado para Bubok (www.bubok.es), y Anboto (www.anbotogroup.com), elegida mejor startup mundial de 2010. Su último libro es Netnografía, sobre investigación social online.

UNED, Madrid, España mdelfresno@der.uned.es

\section{Resumen}

Se presenta una aproximación al estudio de la información disponible en los medios y contenidos generados por los usuarios (MCGU) de la web 2.0. La generación a gran escala de información online es el resultado de una acción social colectiva basada en la información: la infosociabilidad. La inteligencia competitiva (IC) persigue monitorizar e investigar el clima de opinión en la web 2.0 de una organización para generar información relevante para la toma de decisiones. Frente a las posibilidades y limitaciones de la tecnología actual a la hora de procesar la comunicación de significados e ideas abstractas en forma textual, se propone una metodología resultado de investigaciones empíricas realizadas en la web 2.0. Se identifican dos procesos clave: monitorización e investigación, para la generación de insigths orientados a facilitar la toma de decisiones. Se muestra la importancia de cada etapa en relación con los retos metodológicos encontrados extrayendo y analizando gran cantidad de información online.

\section{Palabras clave}

Inteligencia competitiva, Infosociabilidad, Extracción de información, Minería de medios, Minería de textos, Investigación online, Netnografía, Toma de decisiones.

\section{Title: Infosociability: Monitoring and research on the web 2.0 for decision making}

\begin{abstract}
This methodology offers an approach to studying the information available within Web 2.0 Media and User-Generated Content (MUGC). The large-scale generation of online information is the result of collective social action based on information: infosociability. Competitive Intelligence $(\mathrm{Cl})$ aims to monitor and research a company's web 2.0 environment for information relevant to its decision-making process. Facing the possibilities and limitations that today's technology offers for processing the communication of meanings and abstract ideas in text format, a methodology derived from empirical research on web 2.0 is proposed. Monitoring and research are identified as the two key processes that generate insights aimed to facilitate decision-making. The relevance of each stage is illustrated with reference to the diverse methodological challenges encountered while extracting and analyzing large amounts of online information.
\end{abstract}

\section{Keywords}

Competitive intelligence, Infosociability, Information extraction, Media mining, Text mining, Online research, Netnography, Decision making.

Del-Fresno-García, Miguel. "Infosociabilidad: monitorización e investigación en la web 2.0 para la toma de decisiones". El profesional de la información, 2011, septiembre-octubre, v. 20, n. 5, pp. 548-554. 


\section{Introducción}

Aunque en parte es un fenómeno anticipado (Virilo, 1991), la realidad se está transformado a una velocidad nunca antes experimentada debido a su tecnologización, lo que ha acabado por afectar a la forma en que nos comprendemos tanto a nosotros mismos como a nuestra imagen del mundo, de forma individual y colectiva, marcando una clara frontera entre los mundos anterior y posterior a la existencia de internet (Del-Fresno, 2011a, p. 23).

El uso de la Red y del ciberespacio construido sobre ella ha supuesto la reorganización en apenas dos décadas de las costumbres de millones de personas. La utilización de internet alcanzó a inicios de 2011 el $28,7 \%$ de la población mundial $^{1}$, 1.966,5 millones de personas, cuando en 2007 era el 16\%. En la última década el incremento medio de su uso ha sido de un $445 \%$.

Desde la aparición de la Web en la década de los años noventa del siglo pasado han sido impactados muchos ámbitos sociales y organizacionales, como la toma de decisiones empresariales (Citroen, 2011; Xu, 2011), la economía (Ahmed-Sultan, 2011), la expresión de la identidad, la sociabilidad (Del-Fresno, 2011a; Colley; Maltby, 2008), el ocio y la educación formal (Sánchez-Navarro; Aranda, 2011), el activismo (Ahmad-Shaheen, 2008), el aprendizaje (Wen-Yu; Tsai, 2011), y por supuesto el acceso, organización, distribución, consumo, gestión y recuperación de la información (Gopal et al., 2011; Acosta-Valdés et al., 2011; Carrillo-Durán; Nuño-Moral, 2010).

Las redes sociales son el fenómeno emergente más significativo de lo que se denomina web 2.0, que gracias al desarrollo global de código prosocial (Del-Fresno, 2011a, p. 30), el diseño de aplicaciones orientadas a la sociabilidad online (Del-Fresno, 2011a, p. 20), y la eclosión global de la autocomunicación de masas como nueva forma de comunicación en red, ha provocado el aumento de "las oportunidades de cambio social, sin definir por ello el contenido y el objeto de dicho cambio social" (Castells, 2009, p. 29).

Este hecho ha facilitado la producción de una gran cantidad de medios y contenidos por parte de los usuarios (MCGU) (García-de-Torres, 2010; Prescott, 2006) que han entrado en competencia directa con los medios de comunicación tradicionales dando lugar a hibridaciones. Algunos ejemplos son la manera cómo los medios tradicionales han asumido como formato periodístico propio los blogs (Sánchez-Vigil et al., 2010), que se usan como fuentes de información complementaria o alternativa. O el uso de aplicaciones 2.0 para la difusión y acceso no tradicional a la información (Reagan et al., 1998; Salvador; Gutiérrez, 2010). Los medios de comunicación tradicionales se han visto obligados a adaptarse y a realizar cambios en sus redacciones, la tecnología usada, los contenidos o las condiciones de trabajo (Díaz-Noci, 2010; Noguera, 2010). $\because$ QuOr 2.0 Contacto 曾 Vídeos

Webrunner 2.0 ofrece:

RELEVANCIA RAPIDEZ SIMPLICIDAD ECONOMÍA

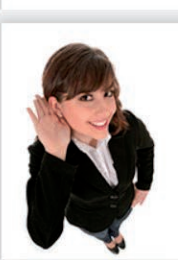

Webrunner 2.o permite:

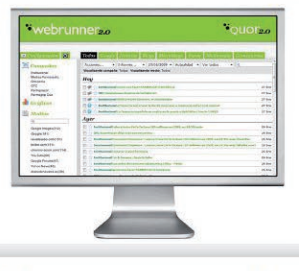

ESCUCHAR IDENTIFICAR VIGILAR

Webrunner 2.0 ha sido elegido como Proyecto Tecnológico Innovado dentro del Plan Avanza 2009

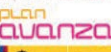
는

Figura 1. Webrunner 2.0 es un programa que permite hacer press clippings digitales (monitorización personas, productos...). http://www.webrunner.es

El crecimiento y penetración de los MCGU ha creado un nuevo contexto -global y local al mismo tiempo- para la comunicación, la participación y el intercambio de información. La web social provee a los individuos de plataformas que permiten "mejorar sus capacidades operativas y relaciones" (Del-Fresno, 2011a, p. 46) y facilitan el acceso a informaciones diversificadas desde múltiples fuentes (McLure-Wasko; Faraj, 2005). Parece por tanto apropiado considerar como objeto de estudio el resultado de las interacciones entre la sociabilidad online y la producción y distribución de información en el contexto de la web 2.0, y entender el resultado como una acción social intencional (Cheung; Lee, 2010) colectiva: la infosociabilidad.

En el estudio de la infosociabilidad los sujetos investigados expresan sus opiniones sin tener que ceñirse a cuestionarios estandarizados o guiones

La web 2.0 es un factor acelerador y amplificador de la extraordinaria habilidad de las personas para comunicar significados e ideas abstractas de forma social y colectiva. A pesar de su importancia, existe relativamente poca teoría sobre investigaciones empíricas para comprender este fenómeno de hibridación entre sociabilidad e información y su influencia en la toma de decisiones. La Red está cambiando también el escenario de la investigación "si uno estudia internet como una estructura social o utiliza tecnologías basadas en internet como herramientas para la investigación" (Markham, 2005). La singularidad del ciberespacio estriba en que "evoca, o engendra, maneras de interactuar que antes no eran posibles" (Lessig, 2009, p. 14). El ciberespacio se presenta como un campo de investigación idóneo donde es preciso acceder con una metodología científica tanto para la comprensión de la infosociabilidad como para la elaboración de nuevas teorías o ampliaciones conceptuales de otras ya existentes. 
Lo más característico del estudio de la infosociabilidad para la investigación en general y para la IC en particular, es que los sujetos investigados expresan sus opiniones en la web social sin la obligación de ceñirse a cuestionarios estandarizados o guiones que restrinjan su experiencia a favor de las cuestiones decididas por un investigador.

A continuación se describe un método de investigación -sin ser el único posible- que permite una aproximación natural al objeto de estudio, por lo que ni el trabajo de campo ni los resultados se ven contaminados por la influencia-directa, indirecta, premeditada o casual- del investigador.

Desde el punto de vista de las organizaciones se ha mostrado que existe una correlación entre la investigación en internet y la calidad de la inteligencia competitiva (IC) y también que ésta tiene un impacto positivo en las organizaciones (Teo; Yee-Choo, 2001). La IC es posible extraerla igualmente a partir de las opiniones que los usuarios diseminan en los webs que facilitan que se escriban reseñas y comentarios sobre marcas, productos y servicios de las organizaciones y sus competidores (Xu et al., 2011).

\section{Objetivos y modelo}

Se presenta un modelo metodológico obtenido a partir de investigaciones empíricas realizadas en los últimos años, de extracción y explotación masiva de los MCGU con una herramienta de $\mathrm{IC}^{2}$ en medios sociales de la web 2.0 (figura 2).

Se trata de afrontar con rigor científico los nuevos retos que presenta internet como campo de investigación y, en concreto para el acceso, clasificación, análisis y explotación de la información online generada por los usuarios en los medios sociales. Un modelo de IC para comprender los usos y costumbres de las personas en el ciberespacio y extraer la correspondiente información. Ésta se rastrea o monitoriza, se estructura, analiza, juzga y aplica a la toma de decisiones en un creciente número de ámbitos profesionales y/o organizacionales.

El modelo propone dos horizontes temporales a la hora de abordar una investigación online para IC (figura 3). La información disponible en la web 2.0 se recolecta con Webrunner una aplicación de web mining.

1.La monitorización es la recopilación sistemática de la información delimitada tras seleccionar una serie de keywords o palabras clave estableciendo un corte temporal,

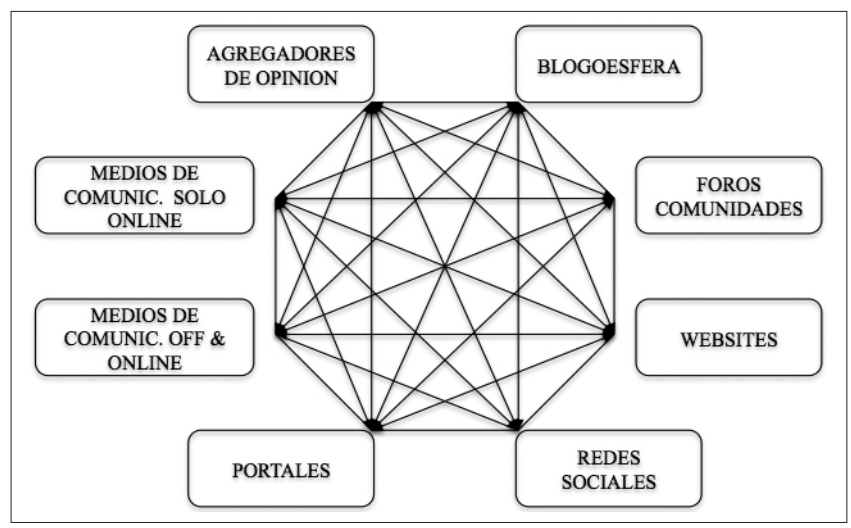

Figura 2. Medios para la monitorización online un hipotético hoy, y haciendo un control diario y acumulativo de la información.

2. La investigación supone remontarnos hacia atrás en el tiempo entre uno y dos años o simplemente meses en función del volumen de información resultante.

Tanto monitorización como investigación tienen dos fases. La primera es de extracción, y en ella se rastrea la información textual o text mining, aunque también puede hacerse un rastreo multimedia o media mining. La segunda fase es de clasificación automática para la monitorización, y automática y manual para la investigación, con el objetivo de no perder la sutileza de las ideas abstractas y los aspectos simbólicos inferibles del lenguaje no analizable por la tecnología.

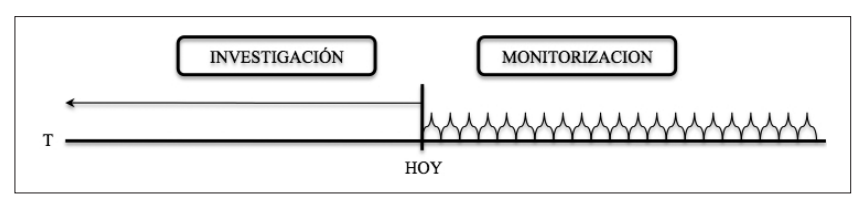

Figura 3. Modelo conceptual para la investigación online

\section{Monitorización}

Una máquina que pudiese entender el lenguaje natural ha sido y es uno de los sueños de la inteligencia artificial desde los mismos orígenes de las ciencias computacionales (Ramsay, 2006). El análisis automatizado de la información en internet, en especial a partir de la emergencia de la web 2.0, se ha convertido en una nueva área de estudio en constante desarrollo. Bajo la denominación de minería web o web mining se engloba todo un conjunto de técnicas encaminadas a la extracción de la información no trivial que reside de manera implícita en los datos. El web mining se usa para identificar y capturar información sobre una temática, marcas, productos o servicios de organizaciones y competidores; y en un sentido más amplio para realizar búsquedas más inteligentes (Landauer, 1998; Larsen, 2002).

La información disponible en la Red es multimedia: texto, sonido, imágenes y bases de datos, pero el modelo presentado aquí se centra en la minería de datos textuales, que hoy son los dominantes.

El valor de la tecnología para la IC está limitado por: a) el estado del saber en cada momento del procesamiento de lenguaje natural (Dedek et al., 2011) y b) la disponibilidad y acceso a tecnologías semánticas (Stumme et al., 2006). Un ejemplo de limitación es la del mismo Google, que siendo el buscador más usado, con una cuota de mercado de alrededor del $90 \%{ }^{3}$, está lejos de ser un buscador sintáctico, lo que condiciona la forma en cómo los usuarios buscamos y accedemos a la información.

La monitorización (figura 4) consiste en la definición y creación de una estrategia de búsqueda y rastreo web, esto es, el establecimiento de un patrón de reconocimiento basado en las características del texto e independiente del tipo de medio online donde se encuentre. Es necesario establecer filtros, reglas de inclusión y exclusión de los resultados, que extraigan información propia y significativa de la temática 


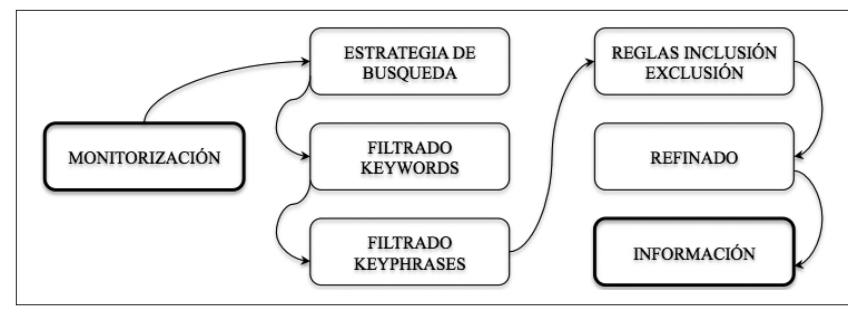

Figura 4. Modelo de proceso para la monitorización online

objetivo. Por último es necesario revisar, ajustar y refinar las palabras clave y las reglas definidas en el inicio de la monitorización, para obtener un universo de datos lo más relevante posible. El sistema puede funcionar de forma automática y continua, sin intervención manual y sin elevados conocimientos lingüísticos.

\section{Investigación}

El web mining semántico en la web 2.0 está también condicionado por el desarrollo de la estructura, arquitectura y posibilidades de navegación semántica de internet, cosa que depende de la aceptación y difusión de los estándares propuestos en el proyecto Semantic Web del W3 $C^{4}$. Conforme se vayan superando estas limitaciones tendrá más sentido hablar de la web 3.0 como convergencia entre la sociabilidad online y la web semántica (Gruber, 2008) y se maximizará su utilidad para la IC.

La mayor limitación en internet no reside por tanto en el acceso al contenido sino a su sentido y significados, ya que como hemos mencionado la tecnología no puede dar una respuesta plenamente satisfactoria. El alcance retrospectivo a abarcar en cada investigación de IC dependerá del propósito de la misma y del volumen de resultados disponibles. Al inicio de la investigación se sigue la misma estrategia de web mining que en la monitorización (figura 4) incorporando un proceso específico (figura 5).

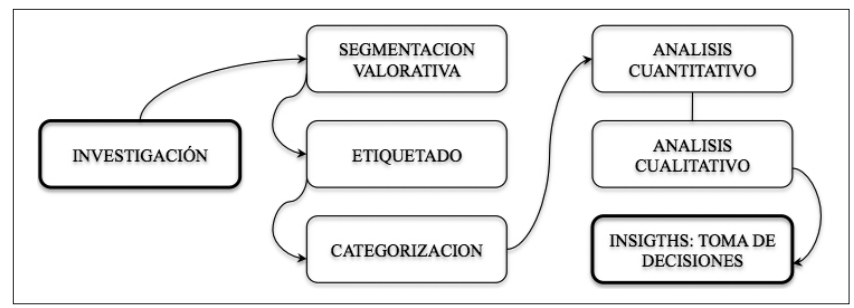

Figura 5. Modelo de proceso para la investigación online

Tras el web mining retrospectivo se dispone de los datos, referencias, que conforman el universo. En caso necesario debido a su volumen puede ser oportuna la selección de una muestra tanto para la fase cuantitativa como la cualitativa. Una referencia es la unidad básica de publicación de información (una noticia en un medio de comunicación online, un comentario en un foro, un post en un blog, etc.) extraída de la monitorización, en formato texto, que contiene al menos una de las palabras clave que delimitan el objeto de investigación desde un punto de vista sintáctico. Las referencias pueden ser limpias o falsos positivos. Una referencia limpia es todo bloque de información extraído de la monitorización en formato texto, relevante para la investigación desde el punto de vista sintáctico y semántico; todas las referencias que no cumplan esta condición son referencias no válidas o falsos positivos.

Como ejemplo, en una investigación de IC realizada sobre medicamentos genéricos (Del-Fresno, 2011b) se eligieron las siguientes palabras clave para la monitorización online: medicamentos genéricos, fármacos genéricos, medicamento $E F G, E F G$ y EFG's. El alcance temporal fueron los años 2009 y 2010 y los idiomas de rastreo español, catalán, gallego y euskera, limitando el alcance geográfico a España. El universo identificado fue de 131.862 referencias limpias.

\subsection{Investigación cuantitativa}

Las referencias se pueden desagregar en opiniones. Una opinión es la unidad mínima de análisis informacional textual significativa siempre que contenga elementos informativos y/o valorativos relevantes para el objeto de estudio. En el ejemplo mencionado se realizó un muestreo aleatorio simple, constituyéndose los conglomerados de referencias en base a la fecha de creación de la referencia. El tamaño final de referencias limpias de la muestra fue $n=339$. La muestra reportaba para el total de referencias un nivel de fiabilidad $\mathrm{e}= \pm 4,5 \%$ con un nivel de confianza del $95,5 \%, 2 \sigma$ respecto de la $\mu$. A partir de las 339 referencias se identificaron 2.406 opiniones (una media de 7,09 opiniones por referencia). La muestra reportó para el total de opiniones un nivel de fiabilidad e $= \pm 2,4 \%$ con un nivel de confianza del 95,5\%, $2 \sigma$ respecto de la $\mu$.

Para poder explotar la información es necesario crear una base de datos. Cada referencia y cada opinión se etiqueta o codifica en función de las siguientes parámetros:

- Idioma/s

- Origen geográfico

- Fecha de creación

- Nombre y url del medio

- Tipo de medio: dónde se ha generado por cada opinante la referencia infotextual (figura 2)

- Tipo de opinante, según su rol: padre, consumidor, trabajador, profesor, potencial usuario, etc.; los roles son dinámicos.

- Tipo de opiniones

- Tema

- Valoración temática

- Actitud del opinante: carga actitudinal o sentiment del opinante.

- Identidad del opinante: anonimato, pseudonimato, transparente.

Una vez codificadas las referencias y opiniones se diseña un plan de explotación ad hoc y se genera información estadística para dimensionar bajo parámetros científicos los resultados de la investigación.

\subsection{Investigación cualitativa}

Tras la fase cuantitativa el modelo propone una fase cualitativa (figura 4), que permite conocer una parcela de la realidad, aunque en nuestro caso tal realidad esté en el ciberespacio. En las sociedades modernas gran parte de la vida social está descrita por textos escritos de muy diferentes formas (Atkinson; Coffey, 1997) y, en muchos casos, los 
investigadores que "usan textos escritos no siguen ningún protocolo definido al hacer su análisis. Por medio de lecturas y relecturas de sus materiales empíricos tratan de precisar los temas y, en consecuencia, dibujar una imagen de las presuposiciones y significados que conforman el mundo cultural del que el material textual es altamente representativo" (Peräkylä, 2005).

Se trata de analizar las referencias para generar insights (percepciones, puntos de vista) útiles para la toma de decisiones (Chase, 2005). La información extraída expresa el pensamiento de los sujetos que crean los contenidos de forma voluntaria y con objetivos no necesariamente concurrentes. El contexto social online permite al investigador acceder a una narrativa informacional total, recuperada y presentada como un todo, retrospectiva, naturalista y plena de significado.

Gracias al fenómeno de la sociabilidad online, internet tiene "el potencial para cambiar la manera en que los investigadores cualitativos recogen, dan sentido y representan los datos" (Markham, 2005, p. 794), cómo se extrae información y cómo a partir de ella se puede apoyar la toma de decisiones. Para esta fase de la IC el método se deberá elegir en función de los objetivos de la investigación y se optará por una o diferentes aproximaciones dentro de las amplias posibilidades que ofrece la revolución cualitativa (Denzin; Lincoln, 2005).

\section{Discusión}

Si bien internet ha sido un objeto de estudio más propio de las ciencias computacionales y las telecomunicaciones (redes, estándares, protocolos, etc.), las ciencias sociales no pueden considerar el contexto social online como algo ajeno a su objetivo y praxis. Gracias al desarrollo de plataformas diseñadas bajo código prosocial propio de la web 2.0, y al fenómeno de la autocomunicación de masas, ahora se tiene una nueva alternativa de producción, distribución y acceso a la información como nunca había sido posible, al haber desaparecido muchas restricciones geográficas y temporales. Los usuarios de la Red le están otorgando pleno sentido a su desenvolvimiento y uso cotidiano, adaptándola con normalidad a sus vidas. Navegan por el ciberespacio generando una suerte de ecosistema de participación y expresión, lo que plantea nuevos retos para la investigación y comprensión de la acción social colectiva basada en la información: la infosociabilidad.

La recuperación y explotación de la información de la web 2.0 y la generación de nuevo conocimiento para la toma de decisiones depende del estado del arte tecnológico y del refinamiento metodológico para la localización, identificación, recuperación, procesamiento y la explotación inteligente de la información.

Debido a las restricciones semánticas de la tecnología, el análisis debe ser llevado a cabo por parte del investigador mediante procesos de trabajo intensivos. Así, la fase de codificación requiere una interpretación manual que demanda tiempo y energía; y en la fase cualitativa el resultado depende también de las capacidades y conocimientos del investigador. Existe todavía una gran distancia entre la ex- traordinaria habilidad de las personas para comunicar significados e ideas abstractas, y la creación de una máquina que pueda procesar con precisión el lenguaje natural.

El modelo propuesto abre un nuevo campo de investigación para la IC al tener acceso desde una perspectiva científica a los MCGU y la expresión natural de las personas sobre organizaciones, marcas, productos, servicios, competidores, etc.

Desde un punto de vista organizacional, el modelo planteado abre nuevas vías para el desarrollo de la IC gracias a la posibilidad de la identificación temprana de riesgos y oportunidades potenciales por medio de la extracción, recolección, clasificación y análisis de información sobre el clima de opinión de marcas, productos y servicios para dar apoyo a los responsables de la toma de decisiones.

La propuesta de este artículo no era la de identificar leyes universales con la intención de reducir una incertidumbre ontológica, sino la de afinar las herramientas intelectuales para captar, describir, analizar, comprender y explicar mejor la multiplicidad de complejas estructuras de sentido y significación en las que nos hallamos inmersos.

Esto es, desde bases científicas captar el carácter de la organización, generación y difusión de la información en internet para contribuir a reducir parte del enigma de la imbricación entre lo social y lo informacional. En definitiva, para poder comprendernos mejor como individuos y sociedad.

\section{Notas}

1. Internet world stats. Internet usage statistics. World internet users and population stats.

http://www.internetworldstats.com/stats.htm

2. Webrunner es un proyecto tecnológico financiado por el Plan Avanza 2 (Avanza I+D) dentro del área de actuación de Economía Digital.

http://www.webrunner.es

3. http://gs.statcounter.com/\#search_engine-ww-monthly201003-201103

\section{4. http://www.w3.org/2001/sw/wiki/Main_Page}

\section{Referencias bibliográficas}

Acosta-Valdés, Misleiny; Ramírez-Céspedes, Zulia; Manero-Santana, Liliam. "Organización de la información en sitios web periodísticos". El profesional de la información, 2011, enero-febrero, v. 20, n. 1, pp. 54-60.

http://eprints.rclis.org/handle/10760/15756

http://dx.doi.org/10.3145/epi.2011.ene.07

Ahmad-Shaheen, Maqsood. "Use of social networks and information seeking behavior of students during political crises in Pakistan: A case study". The international information \& library review, 2008, Sept., v. 40, n. 3, pp. 142-147. http://dx.doi.org/10.1016/j.iilr.2008.07.006

Ahmed-Sultan, Nabil. "Reaching for the 'cloud': How SMEs can manage". International journal of information management, 2011, June, v. 31, n. 3, pp. 272-278.

http://dx.doi.org/10.1016/j.ijinfomgt.2010.08.001 
Atkinson, Paul; Coffey, Amanda. "Analysing documentary realities". En: Silverman, David (ed.). Qualitative research: theory, method and practice, London: Sage, 1997, pp. 45-62.

Carrillo-Durán, María-Victoria; Nuño-Moral, María-Victoria. "La documentación en la evaluación y gestión de la imagen corporativa". El profesional de la información, 2010, marzo-abril, v. 19, n. 2, pp.123-132.

http://dx.doi.org/10.3145/epi.2010.mar.02

Castells, Manuel. Comunicación y poder. Madrid: Alianza editorial, 2009, 680 p. ISBN 978-8420684994.

Chase, Susan E. "Multiple lenses, approaches, voices". En: Denzin, Norman K.; Lincoln, Yvonna S. (ed.), Handbook of qualitative research, London: Sage, 2005.

Cheung, Christy M. K.; Lee, Matthew K. O. "A theoretical model of intentional social action in online social networks". Decision support systems, 2010, v. 49, n. 1, pp. 24-30. http://dx.doi.org/10.1016/j.dss.2009.12.006

Citroen, Charles L. "The role of information in strategic decision-making". International journal of information management. In press, corrected proof, available online 11 March 2011.

http://dx.doi.org/10.1016/j.ijinfomgt.2011.02.005

Colley, Ann; Maltby, John. "Impact of the internet on our lives: male and female personal perspectives". Computers in human behavior, 2008, Sept., v. 24, n. 5, pp. 2005-2013. http://dx.doi.org/10.1016/j.chb.2007.09.002

Dedek, Jan et al. "Fuzzy ILP Classification of web reports after linguistic text mining". Information processing and management. In press, corrected proof, available online 12 March 2011.

Del-Fresno, Miguel. Netnografía. Barcelona: UOC, 2011(a), 112 p. ISBN 978-8497883856.

Del-Fresno, Miguel. Los medicamentos genéricos como herramienta para el acceso social a la salud. Marcos lingüísticos y creación de clima de opinión online: el caso español. No publicado, en proceso de evaluación, febrero 2011(b).

Denzin, Norman K.; Lincoln, Yvonna S. "The discipline and practice of qualitative research". En: Denzin, Norman K.; Lincoln, Yvonna S. (ed.), The Sage handbook of qualitative research, $3^{\text {rd }}$ ed. London: Sage, 2005, pp. 1-41.

Díaz-Noci, Javier. "Medios de comunicación en internet: algunas tendencias". El profesional de la información, 2010, nov.-dic., v. 19, n. 6, pp. 561-567.

http://www.elprofesionaldelainformacion.com/conteni dos/2010/noviembre/medios_comunicacion.pdf

http://dx.doi.org/10.3145/epi.2010.nov.01

Freire, Juan. "Redes sociales: ¿modelos organizativos o servicios digitales?". El profesional de la información, 2008, nov.-dic., v. 17, n. 6, pp. 585-588.

http://dx.doi.org/10.3145/epi.2008.nov.01

García-de-Torres, Elvira. "Contenido generado por el usuario: aproximación al estado de la cuestión". El profesional de la información, 2010, nov.-dic., v. 19, n. 6, pp. 585-594. http://dx.doi.org/10.3145/epi.2010.nov.04
Gopal, Ram; Marsden, James R.; Vanthienen, Jan. "Information mining. Reflections on recent advancements and the road ahead in data, text, and media mining". Decision support systems. In press, corrected proof, available online 31 Jan. 2011.

Gruber, Thomas. "Collective knowledge systems: where the social web meets the semantic web". Web semantics: Science, services and agents on the world wide web, v. 6, n. 1, Semantic web and web 2.0, Febr. 2008, pp. 4-13.

http://dx.doi.org/10.1016/j.websem.2007.11.011

Landauer, Thomas K. et al. "Learning human-like knowledge by singular value decomposition: a progress report". Advances in NIPS 10, MIT Press, 1998, pp. 45-51.

Larsen, Jan et al. "Web mining: learning from the world wide web". Computational statistics \& data analysis, 2002, Febr., v. 38, n. 4, pp. 517-532.

http://dx.doi.org/10.1016/S0167-9473(01)00076-7

Lessig, Lawrence. El código 2.0. Madrid: Traficantes de sueños, 2009, 560 p. ISBN 978-8496453388.

McLure-Wasko, M.; Faraj, Samer. "Why should I share? Examining social capital and knowledge contribution in electronic networks of practice. MIS quarterly, 2005, v. 29, n. 1, pp. 35-37.

Markham, Annette N. "The methods, politics, and ethics of representation in online ethnography". En: Denzin, Norman K.; Lincoln, Yvonna S. (ed.), The Sage handbook of qualitative research, third edition, London: Sage, 2005, pp. 793-820.

Noguera-Vivo, José-Manuel. "Panorámica de la convergencia periodística: los caminos hacia la redacción Google". El profesional de la información, 2010, nov.-dic., v. 19, n. 6, pp. 652-657.

http://eprints.rclis.org/handle/10760/15069 http://dx.doi.org/10.3145/epi.2010.nov.12

Peräkylä, Anssi. "Analyzing talk and text". En: Denzin, Norman K.; Lincoln, Yvonna S. (ed.), The Sage handbook of qualitative research, 3rd ed. London: Sage, 2005, 870 pp.

Prescott, LeeAnn. Hitwise US consumer generated media report. 2006.

http://64.89.37.123/whitepapers_docs/CGM\%20Report.pdf

Ramsay, Allan. "Natural language understanding, automatic". En: Keith Brown, editor-in-chief, et al. Encyclopedia of language \& linguistics, Oxford: Elsevier, 2006, pp. 524-539.

Reagan, Joey et al. "Motivations as predictors of information source perceptions: traditional media and new technologies". Telematics and informatics, 1998, Febr., v. 15, n. 1-2, pp. 1-10.

http://dx.doi.org/10.1016/S0736-5853(98)00001-X

Salvador-Benítez, Antonia; Gutiérrez-David, María-EstreIla. "Redes sociales y medios de comunicación: desafíos legales". El profesional de la información, 2010, nov.-dic., v. 19, n. 6, pp. 667-674.

http://dx.doi.org/10.3145/epi.2010.nov.14

Sánchez-Navarro, Jordi; Aranda, Daniel. "Internet como fuente de información para la vida cotidiana de los jóvenes 
españoles". El profesional de la información, 2011, enerofebrero, v. 20, n. 1, pp. 32-37.

http://eprints.rclis.org/bitstream/10760/15332/1/032-037. $p d f$

http://dx.doi.org/10.3145/epi.2011.ene.04

Sánchez-Vigil, Juan-Miguel; Marcos-Recio, Juan-Carlos; Olivera-Zaldua, María. "Presencia y contenidos de los blogs en los principales diarios españoles". El profesional de la información, 2010, nov.-dic., v. 19, n. 6, pp. 602-609. http://dx.doi.org/10.3145/epi.2010.nov.06

Stumme, Gerd; Hotho, Andreas; Berendt, Bettina. "Semantic web mining: State of the art and future directions". Web semantics: Science, services and agents on the world wide web, 2006, June, v. 4, n. 2, pp. 124-143.

http://dx.doi.org/10.1016/j.websem.2006.02.001
Teo, Thompson S. H.; Yee-Choo, Wing. "Assessing the impact of using the Internet for competitive intelligence". Information \& management, 2001, Nov., v. 39, n. 1, pp. 67-83. http://dx.doi.org/10.1016/S0378-7206(01)00080-5

Virilo, Paul. The aesthetics of disappearance. New York: Semiotext(e), 1991, 128 p. ISBN 978-1570270413.

Wen-Yu Lee, Silvia; Tsai, Chin-Chung. "Students' perceptions of collaboration, self-regulated learning, and information seeking in the context of Internet-based learning and traditional learning". Computers in human behavior, 2011, March, v. 27, n. 2, pp. 905-914.

http://dx.doi.org/10.1016/j.chb.2010.11.016

$\mathrm{Xu}$, Kaiquan et al. "Mining comparative opinions from customer reviews for competitive intelligence". Decision support systems, 2011, March, v. 50, n. 4, pp. 743-754. http://dx.doi.org/10.1016/j.dss.2010.08.021

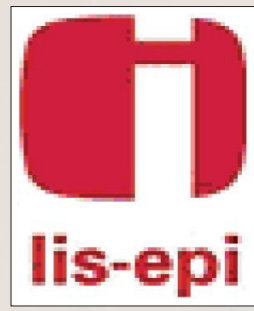

\section{1st international workshop Information transfer for innovation 3 de noviembre de 2011 Universidad Politécnica de Valencia}

\section{Temas:}

Gestión de la información, Innovación y Comunicación científica.

\section{Objetivos:}

- Conocer el estado actual del concepto de contenidos basados en la calidad.

- Mostrar las tendencias en: redes y archivos abiertos; repositorios y archivos; redes sociales; innovación en contenidos, formatos y plataformas.

- Presentar casos reales en el uso de linked data en sistemas de información.

Más información:

http://www.ciepi.org/transferinnovation

\section{Organizan:}

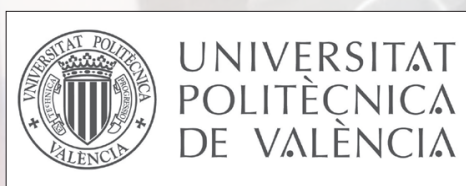

Participan:

El profesional de la

\section{Patrocina:}

CONSEJO SUPERIOR

DE INVESTIGACIONES CIENTÍFICAS

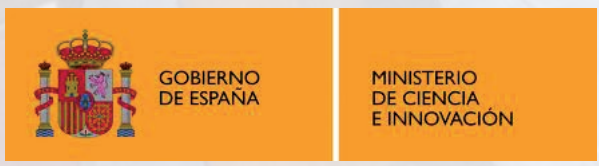

